\title{
TPVs PA/NBR: Sistema de Reticulação e Propriedades
}

\author{
Enio C. M. Fagundes \\ Programa de Pós-Graduação em Ciências dos Materiais, UFRGS \\ Instituto Federal de Educação, Ciência e Tecnologia Sul-rio-grandense, IFSul
}

\author{
Marly A. M. Jacobi \\ Programa de Pós-Graduação em Ciências dos Materiais, UFRGS
}

Resumo: Elastômeros termoplásticos vulcanizados, TPVs, à base de co-poliamida (PA6,6-6) e borracha nitrílica (NBR), foram preparados por vulcanização dinâmica em câmara de mistura a partir de um sistemas de vulcanização tendo como componente principal o peróxido de dicumila e os coagentes bismaleimida e enxofre. Variando-se a proporção dos componentes, estudou-se a influência do enxofre sobre as propriedades mecânicas e deformação permanente à compressão. A morfologia foi determinada por análise de microscopia eletrônica de varredura. Todos os TPVs apresentam propriedades mecânicas superiores à blenda PA/NBR correspondente. TPVs com domínios elastoméricos da ordem de $5 \mu \mathrm{m}$ foram obtidos com o sistema de reticulação constituído de peróxido de dicumila/bismaleimida/enxofre (DCP/BMI/S) na proporção de 2,4/0,4/0,7 php, independentemente da forma de apresentação da NBR (compacta ou pó). Estes materiais apresentaram excelente desempenho mecânico alcançando-se tensões de ruptura da ordem de $20 \mathrm{MPa}$. A presença do enxofre conferiu propriedades mecânicas particulares aos TPVs. Para todos os sistemas de reticulação, os TPVs apresentaram boa resistência química em solventes apolares (iso-octano e óleo 20W50), atribuída à natureza química do par PA/NBR, e valores similares para a deformação permanente à compressão.

Palavras-chave: Elastômero termoplástico vulcanizado, peróxido de dicumila, coagentes de reticulação, morfologia PA/NBR.

\section{PA/NBR TPVs: Crosslink System and Properties}

\begin{abstract}
Thermoplastic vulcanizates (TPVs) based on polyamide (PA6,6-6) and nitrile rubber (NBR) were prepared by dynamic vulcanization in an internal mixer based on a mixed curing system having dicumyl peroxide as the main component and bismaleimide and sulfur as coagents. The proportion of the components as well as the influence of the sulfur content on the mechanical properties and compression set were studied. The morphologies were determined by scanning electronic microscopy. All the TPVs exhibit better properties than the blend. Elastomeric domains of ca. $5 \mu \mathrm{m}$ were obtained for the curing system dicumyl peroxide/bismaleimide/sulfur (DCP/BMI/S) in a proportion of 2.4/0.4/0.7 php, independently of the form of the NBR (crumb or powder). These materials showed excellent mechanical properties, reaching a tension of rupture of about $20 \mathrm{MPa}$. The use of sulfur as curing co-agent gives the material unique properties. All the TPVs exhibited good resistance to non polar solvents (isooctane and oil 20W50) and similar values for the compression set.
\end{abstract}

Keywords: Thermoplastic vulcanizates, crosslinking by dicumyl peroxide, curing coagents, morphology of PA/NBR.

\section{Introdução}

Elastômeros termoplásticos (TPEs) reúnem as propriedades elásticas das borrachas em temperaturas usuais de aplicação com a facilidade de processamento dos polímeros termoplásticos em temperaturas elevadas ${ }^{[1-4]}$. Estas características são consequência da morfologia bifásica decorrente da natureza química diferenciada dos constituintes e do seu processo de obtenção. No caso dos copolímeros constituídos de blocos rígidos e flexíveis e dos termoplásticos olefínicos (TPOs) esta morfologia é definida durante a rota de síntese ${ }^{[5]}$. No caso de elastômeros termoplásticos vulcanizados (TPVs), a morfologia é definida durante o seu processo de obtenção. Depende, essencialmente, da natureza química, da viscosidade, da proporção dos constituintes (termoplástico/borracha), dos agentes de reticulação, da presença ou não de agentes compatibilizantes e do processo de vulcanização dinâmica propriamente dita ${ }^{[5-7]}$. Os agentes de reticulação devem, preferencialmente, atuar sobre a fase elastomérica, provocando uma rápida reticulação, in situ. $\mathrm{O}$ aumento da viscosidade da fase elastomérica, como consequência da reticulação, sob altas taxas de cisalhamento, leva a mesma a se fragmentar, gerando domínios dispersos na fase termoplástica contínua, mesmo esta, estando presente em menor proporção, em massa. A reticulação aumenta a resistência mecânica e química, assim como diminui, significativamente, a deformação permanente a compressão destes materiais se comparados às simples misturas físicas, blendas, correspondentes ${ }^{[8-11]}$. Dadas às características imposta pela matriz termoplástica, os TPVs apresentam as vantagens da processabilidade e da reciclabilidade dos termoplásticos sobre as borrachas termofixas convencionais. Estes são fatores responsáveis pela alta taxa de crescimento anual, entre 6 e $7 \%$, alcançada pelos TPVs na última década, substituindo a borracha termofixa convencional em muitas aplicações, onde perfis complexos são exigidos, como vedações de vidros e portas de carros, recobrimentos de cabos, mangueiras, entre outros ${ }^{[12,13]}$.

Tendo em vista que as propriedades dos TPVs dependem de uma série de fatores que iniciam na escolha da combinação termoplástico/borracha até a otimização das condições de processamento para aquele par, muitos sistemas têm sido explorados desde a sua descoberta por Gessler ${ }^{[14]}$.

Coran e Patel ${ }^{[14]}$ realizaram inúmeros estudos no intuito de obter TPVs com propriedades diferenciadas visando aplicações 
específicas $^{[1,8,15]}$. Da diversidade de sistemas investigados apenas alguns se tornaram comercializáveis, destacando-se os TPVs a base de borrachas de EPM ou EPDM e o termoplástico PP.

Impulsionado pelo mercado automobilístico, materiais com características específicas, de baixo custo de produção, baixa densidade, resistentes a óleos e temperaturas elevadas estão sendo exigidos. Neste particular, poliamidas/ borrachas nitrílicas merecem destaque. Dentro desta classe, Coran et al. ${ }^{[14,16]}$, desenvolveram em câmara de mistura, TPVs a base de poliamidas, como nylon (6, 6-6, 6-9, 10) e borracha nitrílica, NBR, com 33 e $39 \%$ em acrilonitrila, composição 35/65, (em massa). Diferentes agentes reticulantes com destaque ao enxofre, a bismaleimida, a peróxidos e resina fenólica (SP1045) foram aplicados ${ }^{[14]}$. Observaram que, quando temperaturas de processamento superiores a $200{ }^{\circ} \mathrm{C}$ foram utilizadas para a fusão da poliamida um mecanismo de auto-reticulação da NBR pode ocorrer, efeito que contribuiu positivamente para as propriedades finais do TPV. A partir de 2000 destacam-se: Mehrabzadeh e Delfan $^{[17]}$ apresentando TPVs PA-6/NBR (40/60) utilizando diferentes reticulantes como: enxofre, peróxido de dicumila e resina fenólica, obtendo melhor desempenho em termos de inchamento em óleo e propriedades mecânicas para o sistema baseado em resina fenólica. Kumar et al. ${ }^{[18]}$ estudaram o comportamento de fluxo de TPVs a base de PA (6/6-6) e NBR para diferentes proporções de PA/NBR $(70 / 30,50 / 50,30 / 70)$ reticulados com peróxido de dicumila com e sem o uso de polietileno clorado, como agente compatibilizante, obtendo TPVs com domínios elastoméricos muito uniformes. Chowdhury et al. ${ }^{[19,20]}$, descrevem TPVs de PA/ NBR a partir de um terpolímero de poliamida $(6,6-6,6-10)$ e uma NBR carboxilada reticulada com enxofre e aceleradores, obtendo um material com boa resistência em óleo e morfologia com aspecto homogêneo, principalmente para a composição 40/60 de PA/NBR. Gomes et al. ${ }^{[21]}$ estudaram o comportamento sob fluência de TPVs a base de PA6/NBR (28\% acrilonitrila), composição 50/50, com diferentes aditivos, constatando que estes tem grande efeito sobre a fluência destes materiais.

Em um dos estudos realizados por Jacobi et al..$^{[6]}$, mostrou-se que é possível obter, também, TPVs com boa resistência a solventes apolares, a partir de PP cristalino e SBR epoxidada. O inchamento em solventes apolares ficou reduzido em 80\% quando SBR epoxidada em $70 \%$ substituí a SBR normal, comprovando desta forma que é a fase elastomérica a maior responsável pela resistência a solventes, em temperatura ambiente.

Coran et al. ${ }^{[14,16,22]}$ afirmam que a resistência à tração e o alongamento alcançados por TPVs estão relacionados com a sua morfologia. Valores de tensão e alongamento da ordem de $20 \mathrm{MPa}$ e $500 \%$, respectivamente, podem ser alcançados com domínios elastoméricos em torno de $5 \mu \mathrm{m}$. A morfologia é definida durante a vulcanização dinâmica e depende, entre outros fatores, do sistema de cura que, além do agente principal, pode envolver coagentes ${ }^{[1,23,24]}$.

Grimma et al. ${ }^{[25]}$ ao reticular borracha EPDM da forma convencional, com peróxido de dicumila (DCP) combinado com enxofre ou agentes doadores de enxofre, como o 2-mercapto benzotiazol (MBT), obtiveram uma borracha termofixa com alto módulo e alta resistência à ruptura. Observaram, no entanto, que um dos inconvenientes para este sistema, é uma redução na resistência ao envelhecimento da borracha em temperaturas elevadas ${ }^{[26]}$, atribuída as pontes sulfídicas. No entanto, ao introduzirem a bismaleimida (BMI) como terceiro componente, um material com maior resistência à temperatura, sem perda das demais propriedades mecânicas foi obtido ${ }^{[27]}$.

Dado ao sucesso alcançado com este sistema de reticulação na borracha de EPDM, os autores deste trabalho aplicaram sistema similar na vulcanização dinâmica de NBR para à obtenção de TPVs
PA/NBR, visando alcançar resistência a solventes apolares, com destaque a fluidos presentes em automóveis, (combustível e óleos) e a altas temperaturas e um bom desempenho mecânico.

Mesmo com muitos sistemas similares descritos na literatura, a continuidade dos estudos nesta área justifica-se pelos muitos fatores que influenciam as propriedades finais de TPVs. Além disso, na indústria automobilística existe uma grande demanda por TPVs capazes de substituir a borracha termofixa em diferentes setores. Estes devem ter resistência à temperatura, elasticidade, resistência a fluidos, baixa densidade, boa processabilidade e reciclabilidade. Os veículos de passeio são cada vez menores e mais compactos, desenvolvendo maior aquecimento do motor e ambiente periférico, devem ser leves, ter baixo consumo de energia, além de serem ecologicamente corretos (aplicarem materiais recicláveis), exigências estas mais facilmente alcançadas com TPVs do que com borrachas termofixas.

A poliamida 6,6-6 foi escolhida pela sua resistência a temperatura e a NBR pela sua reconhecida resistência a solventes apolares enquanto as demais propriedades como resistência a tração, alongamento, e deformação permanente por compressão, devem ser alcançadas pelo sistema de reticulação. Em estudos anteriores, realizados no nosso laboratório ${ }^{[28,29]}$, foi possível, avaliar a influência de diferentes sistemas de reticulação, como: sistema tradicional enxofre/acelerador, resina fenólica, bismaleimida (BMI), peróxido de dicumila (DCP) e o sistema misto, DCP/BMI/S, sobre as propriedades de TPVs PA/NBR na proporção de 40/60. Constatou-se que o sistema misto resultou em TPVs que exibem excelentes propriedades mecânicas e ótimas resistência a solventes e fluidos apolares.

Dando continuidade aos estudos o presente trabalho descreve a obtenção de TPVs a base de poliamida e NBR, em câmara de mistura, aplicando-se o sistema constituído de peróxido de dicumila e coagentes, com destaque à combinação enxofre e BMI. A influência do teor de enxofre como coagente, assim como a proporção de DCP/BMI no sistema ternário DCP/BMI/S sobre as propriedades finais do TPV como resistência à tração, deformação permanente por compressão, resistência química e morfologia foram avaliadas. Igualmente, a influência da composição PA/NBR e a forma da apresentação física da NBR (compacta e pó) foram estudadas.

\section{Experimental}

Materiais utilizados: co-poliamida PA6/6-6 tipo Radilon CS LX 02066/C NAT (produzida por Radici Group, Brasil), copolímero de acrilonitrila-butadieno com $45 \%$ de acrilonitrila, tipo Thoran 4560 (produzida por Petroflex agora Lanxess, Brasil), copolímero de acrilonitrila-butadieno com 39\% de acrilonitrila, tipo NP 2130 (produzida por Nitriflex, Brasil), peróxido de dicumila, tipo DCP 40 (produzida por Retilox, Brasil), antioxidante, tipo Ionol CP (produzida por Degussa, agora Evonik), enxofre ventilado (produzido por Flexsys, Brasil) e bismaleimida do tipo comercial CHEMAC HVA-2 (produzida por Chemicon, Brasil). As composições estudadas encontram-se descritas na Tabela 1. Destaca-se que na nomenclatura das amostras tem-se o D para DCP; B para o BMI; S para o enxofre, seguido do respectivo teor de DCP empregado de tal forma que a amostra DBS significa reticulada pelo sistema misto: peróxido de dicumila, bismaleimida e enxofre. Quando não indicado, as composições PA/NBR são de 40/60 em massa. Nas amostras contendo NBR na proporção 65 e $70 \%$ houve ajuste nos agentes de cura. As composições são dadas em php (partes por cem de polímero).

$\mathrm{O}$ processamento do TPV foi realizado em câmara de mistura HAAKE RHEOCORD munido de rotores tipo "roller" em 
Fagundes, E. C. M.; Jacobi, M. A. M. - TPVs PA/NBR: sistema de reticulação e propriedades

Tabela 1. Composições processadas com os teores dos componentes em php (partes por cem de polímero). Simbologia utilizada: D = DCP, B = BMI, $\mathrm{S}=$ enxofre.

\begin{tabular}{cccccccccc}
\hline Componentes & Blenda & DBS2.4 & DBS2.6 (35/65) & DBS2.8 (30/70) & DB 2.4_0.4 & DB 2.4_0,8 & DB 3.4_0.6 & DB 3.4_1.1 & DBSpo2.4 \\
\hline NBR & 60,0 & 60,0 & 65,0 & 70,0 & 60,0 & 60,0 & 60,0 & & 60,0 \\
NBR pó & & & & & & & & & \\
PA & 40 & 40,0 & 35,0 & 30,0 & 40,0 & 40,0 & 40,0 & 40,0 & 40,0 \\
(antioxidante) & 1,0 & 1,0 & 1,0 & 1,0 & 1,0 & 1,0 & 1,0 & 1,0 & 1,0 \\
Ionol CP (and, & 2,0 & 2,8 & 2,4 & 2,4 & 3,4 & 3,4 & 2,4 \\
DCP 40 & & 2,4 & 2,6 & 0,5 & 0,4 & 0,8 & 0,6 & 1,1 & 0,4 \\
BMI & & 0,4 & 0,4 & 0,4 & - & - & - & - & 0,7 \\
Enxofre & & 0,7 & 0,8 & 0,8 & - & & & &
\end{tabular}

1) PA é uma co-poliamida 6/6-6 e; 2) DCP 40 possui $40 \%$ de DCP ativo.

duas etapas. Na primeira etapa preparou-se uma pré-mistura da borracha, do antioxidante e coagentes (Tabela 1), em um período de 4 minutos, a $70^{\circ} \mathrm{C}$. Na segunda etapa fundiu-se o termoplástico (2 minutos) a $195{ }^{\circ} \mathrm{C}$, seguindo-se da incorporação da pré-mistura (4 minutos) e a adição e incorporação do sistema de reticulação, ( 2 minutos). O material foi removido da câmara e moldado, por compressão, em placas de $100 \times 100 \times 2 \mathrm{~mm}$ a $195^{\circ} \mathrm{C}$, em prensa mecânica com aquecimento controlado e resfriamento rápido. $\mathrm{Na}$ composição DBSpo 2.4 não houve necessidade da pré-mistura de borracha, pois a borracha em pó incorpora muito bem na matriz termoplástica fundida. Os corpos de prova para as medidas mecânicas foram obtidos em um vazador pneumático, baseado na norma ASTM D 412 $2^{[30] .}$

\section{Ensaio de tração}

$\mathrm{O}$ ensaio de tração foi realizado no equipamento EMIC DL 5000/10000 baseado na norma ASTM D 412 $2^{[30]}$, utilizando corpode-prova tipo C, velocidade de deformação de $500 \mathrm{~mm} / \mathrm{min}$. No mínimo 5 corpos-de-prova foram ensaiados para o cálculo dos valores médios, de módulo a 100 e $200 \%$ de alongamento e a tensão máxima e alongamento na ruptura.

\section{Deformação permanente por compressão}

Os valores de deformação permanente por compressão foram determinados baseado na norma ASTM D $395\left(70 \text { horas } / 70{ }^{\circ} \mathrm{C}\right)^{[31]}$. O corpo-de-prova consiste de dois discos cilíndricos sobrepostos, de dimensões $6.0 \pm 0.2 \mathrm{~mm}$. Este foi deformado até $25 \%$ de sua altura original, usando-se espaçadores para uma medida mais precisa. $\mathrm{O}$ corpo-de-prova foi removido do molde e após 30 minutos mediu-se sua espessura final.

\section{Resistência a solvente apolar - medidas de inchamento}

O grau de inchamento até o equilíbrio foi determinado em solventes apolares (iso-octano e óleo automotivo 20W50). Três amostras de mesma composição foram imersas nos solventes por 72 horas na temperatura de $70^{\circ} \mathrm{C}$ (tempo suficiente para atingir-se o equilíbrio) e a massa da amostra inchada foi determinada, baseada na norma ASTM D 471 $1^{[32]}$. Os resultados são expressos como massa de solvente absorvido por massa de TPV seco.

\section{Avaliação morfológica}

A morfologia foi determinada por microscopia eletrônica de varredura (MEV) realizada no Centro de Microscopia Eletrônica da UFRGS em um equipamento modelo Jeol JSM 6060. As superfícies das amostras cortadas com bisturi foram tratadas com ácido acético glacial por 2 horas $/ 100^{\circ} \mathrm{C}$, com a finalidade de extrair parcialmente a poliamida superficial e facilitar a visualização da fase elastomérica. As amostras secas foram metalizadas com ouro e analisadas.

\section{Resultados e Discussão}

\section{Resistência a tração}

Pela análise qualitativa das placas observa-se que todas as composições são macroscopicamente homogêneas e exibem boa flexibilidade. Os TPVs nos quais foi incluído enxofre no sistema de reticulação apresentaram coloração castanha, típica de enxofre escurecido em função da temperatura de processamento aplicada. A Figura 1 apresenta as curvas medianas de tensão-deformação de todos os TPVs descritos na Tabela 1. As amostras de PA/NBR, denominadas DBS 2.6 e DBS 2.8 apresentam, respectivamente, 65 e $70 \%$ de NBR em sua composição, sendo que o teor de DCP e coagentes foi ajustado em função da mesma. Os resultados mostram que tanto o sistema de cura, presença ou não de enxofre, teor de DCP/BMI, quanto a composição exercem uma influência sobre as propriedades mecânicas, de maneira similar ao descrito na literatura ${ }^{[33]}$. A partir destas curvas, avaliou-se a tensão máxima, o módulo a 100 e $200 \%$, assim como o alongamento na ruptura.

Observa-se que a mistura física PA/NBR (blenda) apresenta propriedades mecânicas muito pobres se comparada às demais composições de PA/NBR reticuladas (TPVs) como usualmente observado $^{[34]}$. A composição DBS 2.4 (PA/NBR 40/60) reticulada com o sistema peróxido de dicumila, BMI e enxofre na proporção DBS 2,4/0,4/0,7 destaca-se das demais, no que tange seus valores de tensão máxima de aproximadamente $20 \mathrm{MPa}$ e alongamento na ruptura próxima a $300 \%$, valores estes, considerados excelentes, para perfomance de $\mathrm{TPVs}^{[14,16,17]}$.

Esta tensão deve ser atribuída à contribuição da poliamida, $\mathrm{PA}$, e a morfologia da fase elastomérica alcançada com este sistema de reticulação, constituído de domínios da ordem de $5 \mu \mathrm{m}$, homogeneamente distribuídos na fase termoplástica, conforme pode ser visto na Figura 4d, a ser discutida em detalhes no item morfologia. Atribui-se o alongamento elevado ao efeito dos coagentes no sistema de reticulação (BMI e enxofre) visto que o DCP é reconhecido como um agente de reticulação eficiente, mas que resulta em reticulados que apresentam baixa deformação ${ }^{[35]}$. Da mesma forma os valores de módulo tendem a acompanhar a performance da tensão máxima, com valores de 13,0 e 16,5 $\mathrm{MPa}$ para 100 e $200 \%$ de deformação, respectivamente.

O comportamento diferenciado do TPVs DBS 2.6 e DBS 2.8 podem ser atribuídos a sua composição (maior teor de NBR, 65 e $70 \%$ em massa, respectivamente) e consequentemente a morfologia, decorrente desta composição. O TPV DBS 2.6 apresentou menor valor no alongamento, assim como, menor tensão de ruptura, na ordem de 13,6 MPa. As propriedades da amostra DBS 2.8 foram bem inferiores, apresentando uma tensão máxima de $12,2 \mathrm{MPa}$, e alongamento de $255 \%$.

A importância do enxofre, como coagente no sistema de reticulação foi avaliada nas composições, DB 2.4_0.4, DB 2.4_0.8, 
DB 3.4_0.6, DB 3.4_ 1.1, formuladas apenas com DCP e BMI. Enquanto no TPV DB 2.4_0.4 simplesmente o enxofre foi eliminado, nas demais o seu teor foi compensado pelo aumento de DCP (DB 3.4_0.6 e DB 3.4_1.1) ou BMI (DB 2.4_0.8). Todas as composições caracterizam-se por apresentarem propriedades mecânicas como: tensão máxima, módulo e alongamento, inferiores ao do sistema DBS. A importância da presença do enxofre pode ser constatada ao se comparar as composições DBS 2.4 e DB 2.4_0.4, esta sem enxofre, e propriedades muito inferiores, apesar de apresentarem mesmo teor de DCP e BMI. A amostra DB 2.4_0.8 teve seu teor de enxofre compensado pelo aumento do BMI apresentando uma performance bem inferior. Da mesma forma um aumento do DCP (DB 3.4_0.6) não resultou em propriedades similares. Só o aumento significativo do DCP combinado com o aumento no teor de BMI, TPV DB 3.4_1.1 resultou em um material com bom desempenho mecânico, apresentando uma tensão de ruptura de 11,4 MPa e alongamento de $175 \%$. Estes resultados estão em concordância com os publicados por Grimma et al. ${ }^{[25]}$, onde o mesmo abordou a importância do enxofre e BMI na reticulação da borracha termofixa de EPDM com peróxido de dicumila.

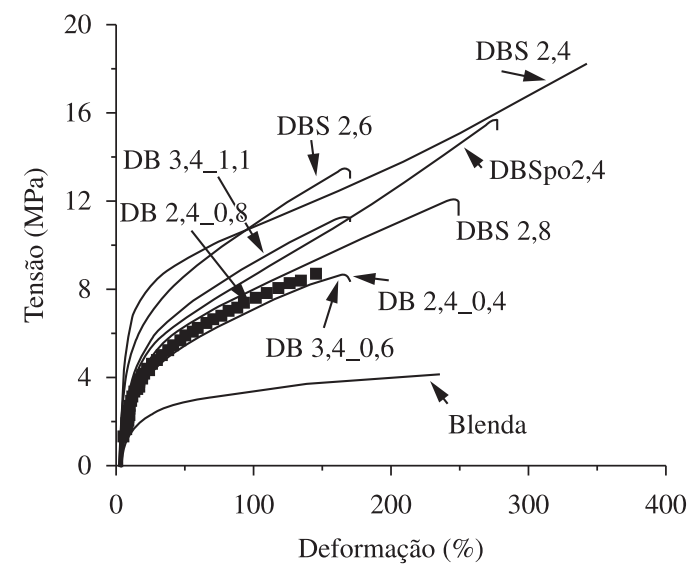

Figura 1. Curva tensão-deformação da blenda PA/NBR 40/60 e dos TPVs.
A composição DBSpo 2.4 apresentou bons resultados alcançando para a tensão máxima $15,8 \mathrm{MPa}$ e alongamento de $246 \%$, exibindo uma performance pouco inferior apenas em relação a composição DBS2.4. Ressalta-se que a diferença deste TPV em relação ao TPV DBS 2.4 é na forma de apresentação da borracha (forma de pó) e no teor de acrilonitrila, de 39\%, inferior ao da amostra DBS 2.4 que é de $45 \%$. A vantagem da composição DBSpo 2.4 é o ganho de tempo e agilidade no processo de preparação da mistura que envolve apenas uma etapa. Com esta composição houve também um ganho em termos da facilidade de descarregamento da câmara de mistura, uma vez que a presença da carga CaCO3, na NBR em pó, facilita o descolamento da composição do interior da câmara e dos rotores, tarefa bastante difícil quando realizada com a borracha em fardo, utilizada nas demais composições. Deve ser salientado que a NBR em pó leva grande vantagem em um processamento contínuo como extrusão, no qual a borracha só poderá ser alimentada na forma de pó ou granulada. No nosso estudo, atribuímos a alta performance alcançada pelas amostras DBS 2.4 (compacta e pó) a morfologia alcançada por estes TPVs, assim como a natureza diferenciada dos pontos de reticulação da fase elastomérica ${ }^{[36]}$. Enquanto, a reticulação com peróxido gera ligações cruzadas carbono-carbono, pouco flexíveis, a reticulação com enxofre e com BMI pode gerar interligações mais longas, portanto, mais flexíveis.

\section{Deformação Permanente a Compressão - DPC}

A deformação permanente a compressão determina o grau de deformabilidade residual que o material apresenta após ser submetido a uma carga compressiva prolongada ${ }^{[30]}$, importante propriedade em aplicações industriais. Uma das desvantagens dos TPVs em relação às borrachas termofixas convencionais é que apresentam valores de DPC bem superiores, devido à forte influência da matriz termoplástica que se deforma plasticamente sob compressão. Os valores de DPC obtidos para os TPVs são exibidos na Figura 2. Todos os TPVs apresentam melhor

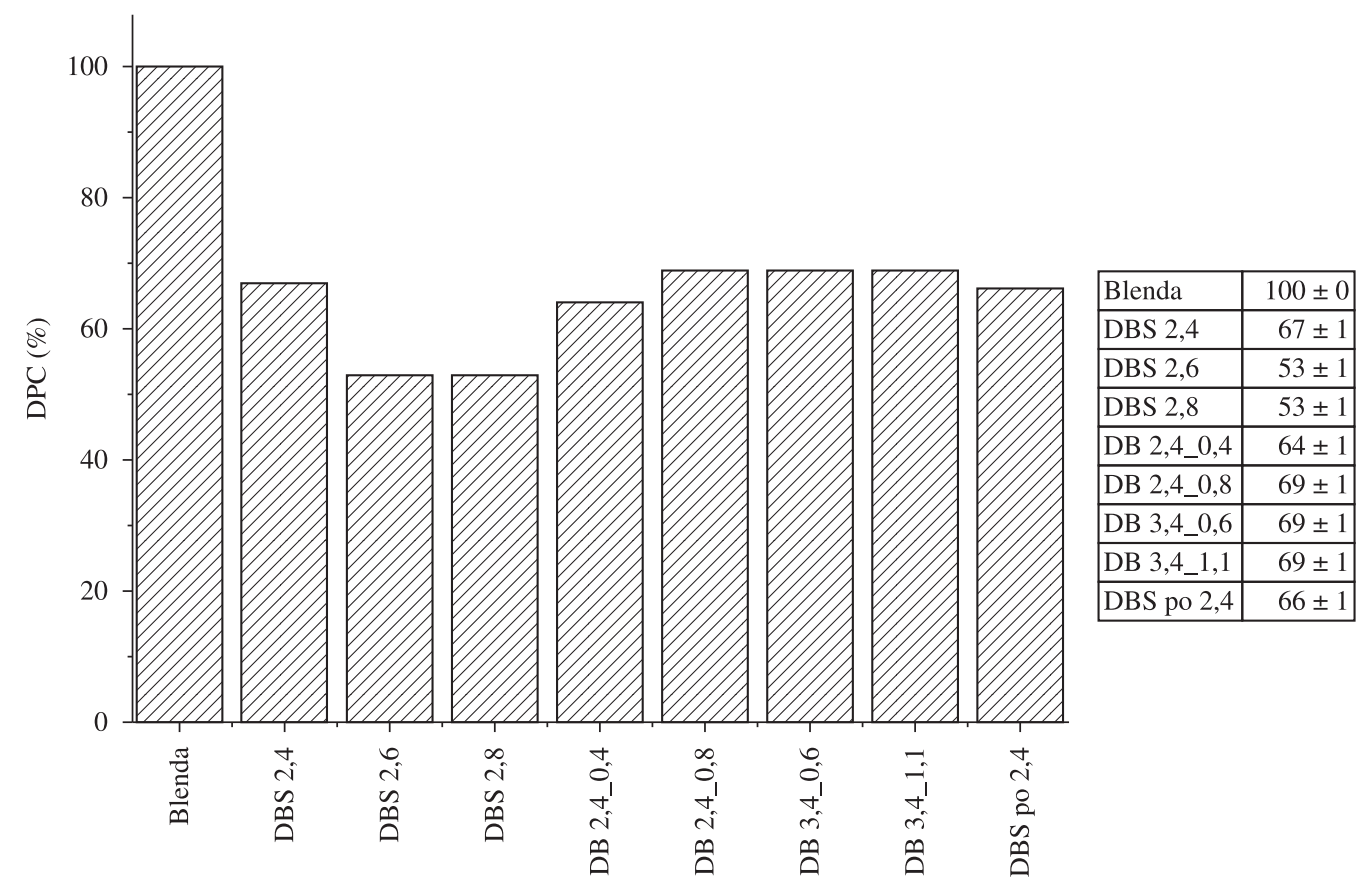

Figura 2. Deformação permanente à compressão dos TPVs com os respectivos desvios padrão. 
desempenho que a blenda, com praticamente, o mesmo valor, para as composições 40/60, independente do sistema de cura, e um valor levemente inferior para as composições 35/65 (DBS 2.6) e 30/70 (DBS 2.8) confirmando que a reticulação e o teor da fase elastomérica contribui de forma positiva para o alcance de melhor performance ${ }^{[37,38]}$ e que a responsabilidade do fraco desempenho dos TPVs, comparado às borrachas convencionais, deve-se a matriz termoplástica que se deforma, de maneira irreversível, sob compressão.

\section{Resistência a solventes}

Lembrando que um dos objetivos da obtenção de TPVs a base de PA/NBR é a sua resistência, em potencial, a solventes orgânicos apolares, esta propriedade foi avaliada por inchamento em dois solventes considerados bastante apolares, iso-octano e óleo de motor 20W50. Os resultados encontram-se na Figura 3, onde valores negativos indicam pequena perda de massa durante a medida como consequência da extração de aditivos e ou produtos oriundos da reticulação e polimerização. A NBR sintetizada em emulsão deve conter produtos residuais de sua síntese, como emulsificantes. O inchamento máximo alcançado independente do solvente foi de aproximadamente, 1,5\% (em massa), um valor extremamente pequeno, indicando que estes materiais não possuem afinidade por compostos apolares e, portanto, são resistentes aos mesmos. Este comportamento pode ser atribuído, essencialmente, a natureza química dos polímeros que constituem o TPV (PA/NBR), pois os valores da blenda encontram-se na mesma ordem de grandeza.

\section{Morfologia - MEV}

A avaliação morfológica é importante para compreender e relacionar a microestrutura às propriedades macroscópicas do material. Coran \& Patel ${ }^{[14,39,40]}$ observaram que quanto menor o tamanho dos domínios da fase elastomérica dispersa na matriz plástica, melhor é o desempenho mecânico do TPV. A morfologia é definida durante o processo da vulcanização dinâmica e depende da natureza química e compatibilidade dos componentes da mistura, da eficiência da reticulação e da metodologia do processo de mistura. Mesmo sendo a borracha a maior fração em massa, esta ao ser reticulada dinamicamente, na presença do plástico fundido, torna-se a fase dispersa ${ }^{[11-43]}$. Micrografias obtidas por microscopia eletrônica de varredura, MEV, de superfícies devidamente preparadas dos TPVs DBS 2.8, DBS 2.6, DBSpo 2.4 e DBS 2.4 são apresentadas na Figura 4. A análise qualitativa nos permite afirmar que as amostras DBS 2.4 e DBSpo 2.4 apresentam domínios de NBR bem definidos, aproximadamente esféricos, homogeneamente dispersos na matriz de PA. A amostra DBS 2.8 (30/70) apresenta morfologia cocontínua para a fase elastomérica, indicando que não ocorreu uma completa inversão de fase, o que deve ser atribuído ao alto teor de $\mathrm{NBR}^{[23]}$. No caso do TPV DBS 2.6 (35/65) observam-se domínios elastoméricos não muito bem definidos. A composição tem influência sobre o fenômeno conhecido como inversão de fase ${ }^{[41-44]}$ e portanto sobre a morfologia.

O tamanho médio dos domínios elastoméricos foi determinado utilizando-se o software livre Image J versão 4.41 o (Wayne Rasband - National Institute of Health - USA) apresentado no histograma da Figura 5, resultando em valores médios de $4.3 \pm 0,9 \mu \mathrm{m}$ para o TPV DBS 2.4 e de $5,1 \pm 0,7 \mu \mathrm{m}$, para o TPV DBSpo 2.4, respectivamente. Esta morfologia deve ser responsável pela excelente performance mecânica destes materiais ${ }^{[14,16,17]}$ alcançando-se valores para tensão de ruptura de 20 e $16 \mathrm{MPa}$ respectivamente, similarmente, ao descrito por Coran \& Patel ${ }^{[39,40,45]}$ em seus estudos. Estes autores relacionam o tamanho dos domínios elastoméricos com as propriedades mecânicas encontrando valores de tensão de ruptura de $20 \mathrm{MPa}$ para domínios da ordem de grandeza de $5 \mu \mathrm{m}$.

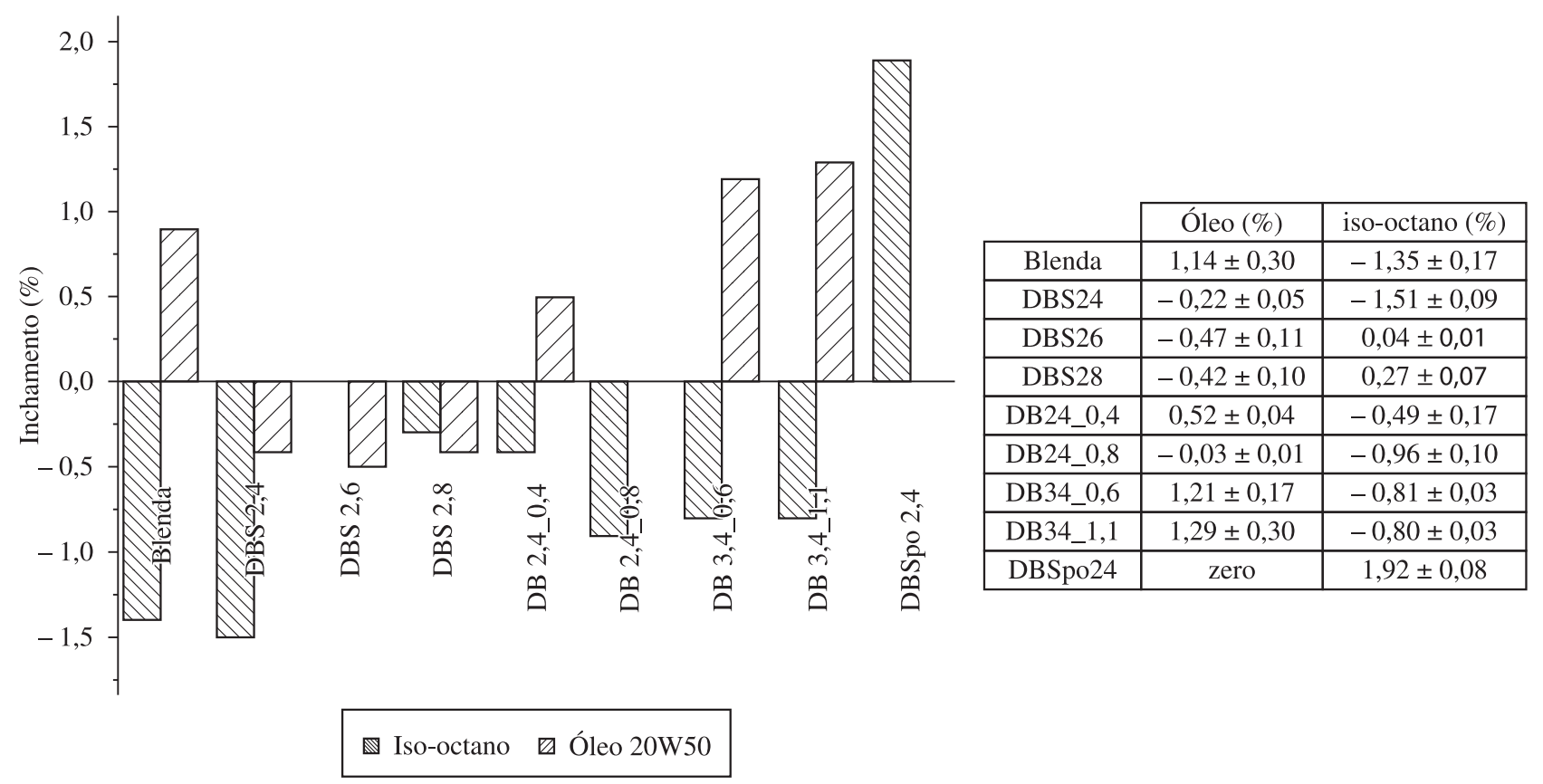

Figura 3. Comportamento dos TPVs em solventes apolares com os respectivos desvios padrão. 


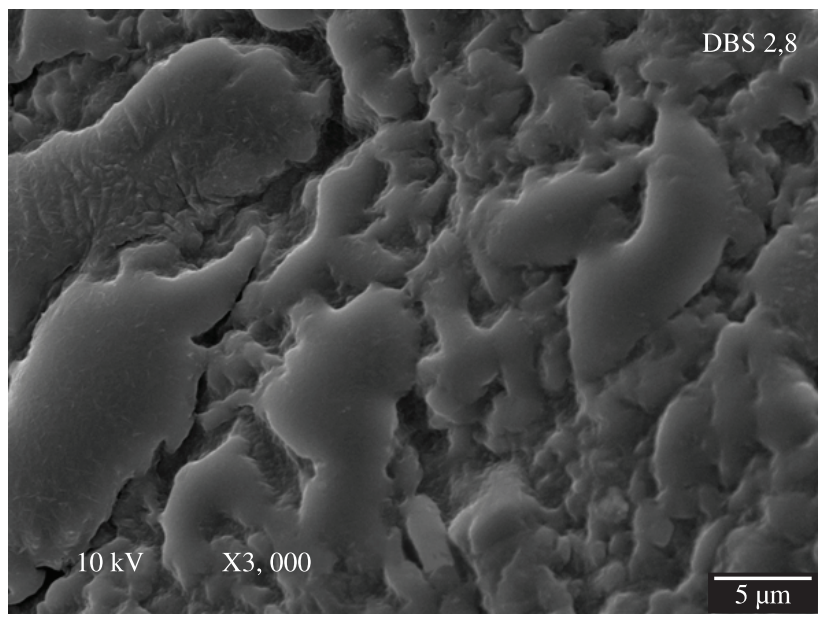

(a)

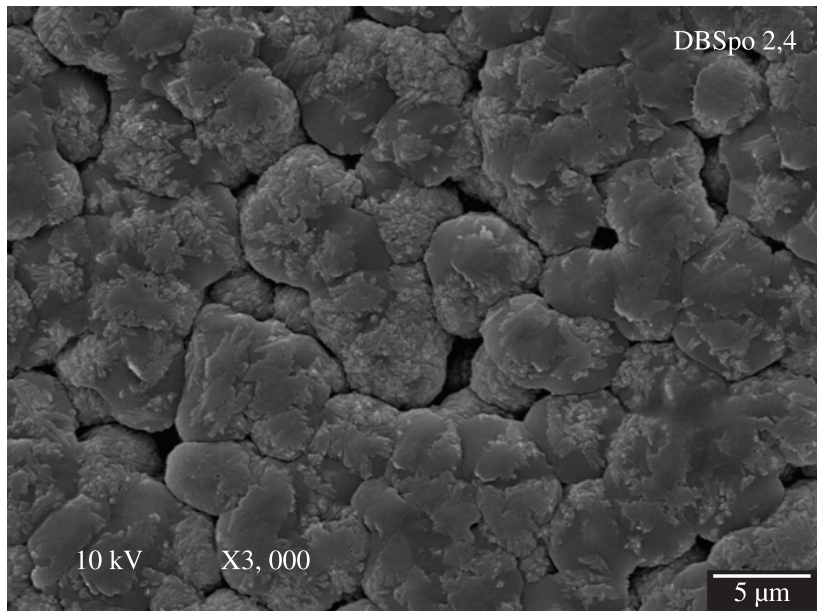

(c)

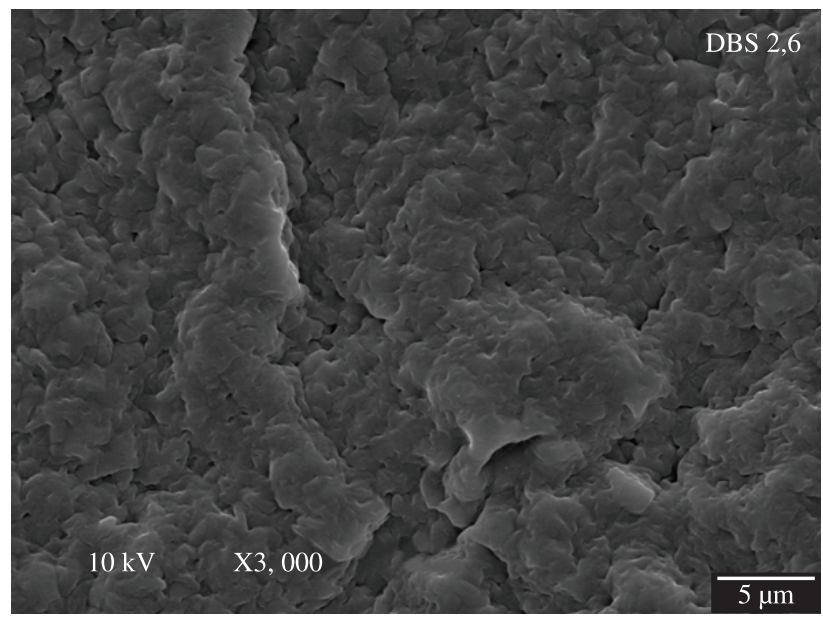

(b)

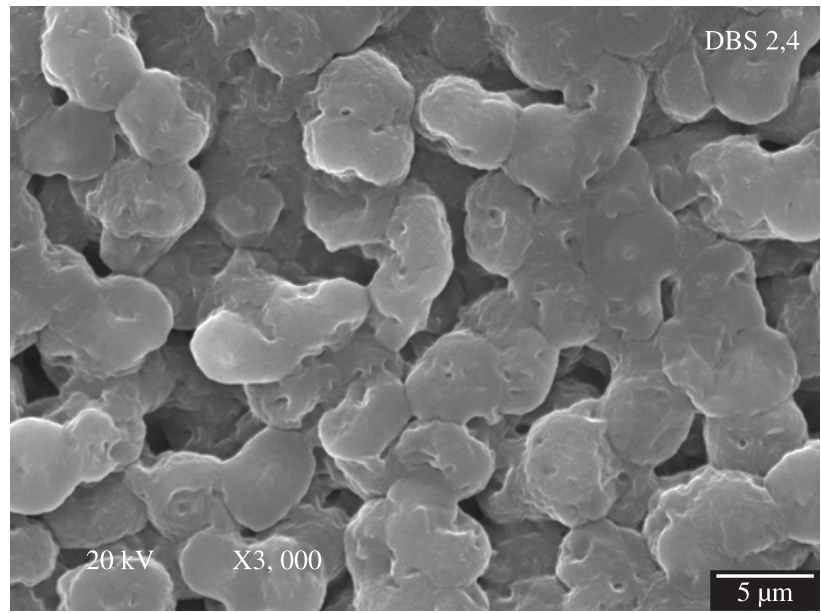

(d)

Figura 4. Micrografias (MEV) dos TPVs a) DBS 2.8; b) DBS 2.6; c) DBSpo 2.4 e; d) DBD 2.4. Aumento de 3000 vezes.

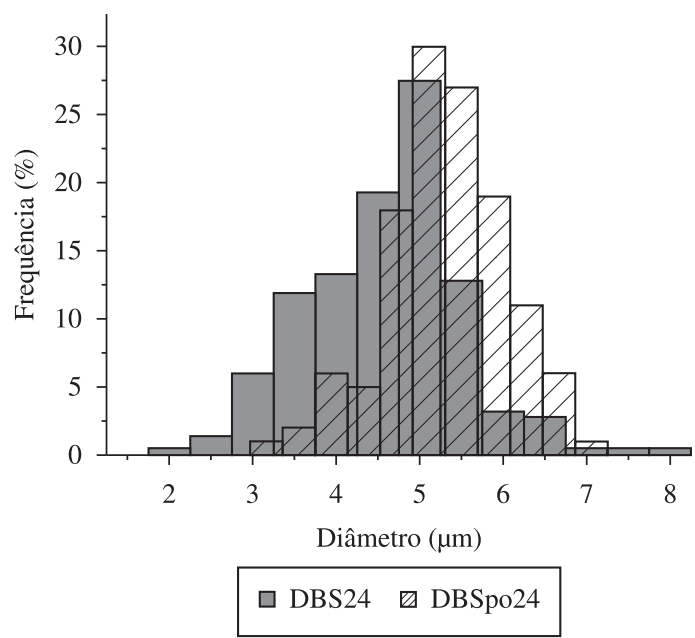

Figura 5. Distribuição dos tamanhos dos domínios elastoméricos na amostra DBS 2.4 e DBSpo 2.4.

\section{Conclusões}

O presente trabalho demonstrou que é possível obter TPVs com excelente desempenho mecânico e resistência a fluidos orgânicos apolares a partir de PA6,6-6/NBR, em composição 40/60, em massa, utilizando-se como agente de reticulação um sistema misto constituído de peróxido de dicumila e coagentes bismaleimida e enxofre. A partir de uma adequada proporção dos agentes, obteve-se uma morfologia com domínios elastoméricos da ordem de $5 \mu \mathrm{m}$, homogeneamente dispersos na matriz termoplástica. Observou-se, também, que as propriedades dos TPVs dependem da proporção PA6,6-6/NBR e que a presença do enxofre como coagente é fundamental para alcançarem-se as altos valores de tensão e alongamento na ruptura. Valores menores de deformação permanente à compreensão são alcançados para teores de NBR de 65 e $70 \%$ em massa, no entanto perde-se em tensão e alongamento na ruptura. Os estudos preliminares apontam que a utilização NBR em forma pó resulta em materiais com propriedades mecânicas levemente inferiores, mas com ganho em processabilidade, o qual compensa grandemente a sua aplicação sempre que possível. Até onde se tem conhecimento este sistema de reticulação não tem sido ainda aplicado na obtenção de TPVs. Os resultados mostram que o sistema aqui desenvolvido tem grande potencial tecnológico, uma vez que tanto os polímeros quanto os componentes do sistema de cura são, comercialmente, acessíveis no mercado e amplamente utilizados na tecnologia das borrachas termofixas. Composições com maiores teores de borracha, apesar do grau de reticulação equivalente da fase elastomérica, apresentam valores de tensão e alongamento na ruptura inferior, mas menor deformação permanente à compressão. 


\section{Agradecimentos}

Os autores agradecem as agências CNPQ e FAPERGS e IFSul pelo apoio financeiro.

\section{Referências Bibliográficas}

1. De Risi, F. R. \& Noordermeer, J. W. M. - Rubber Chem. Technol., 80, p.83 (2007). http://dx.doi.org/10.5254/1.3548170

2. George, J.; Varughese, K. T. \& Thomas, S. - Polymer., 41, p.1507 (2000). http://dx.doi.org/10.1016/S0032-3861(99)00302-X

3. Blume, M. \& Schuster, R. H. - Kautsch. Gummi Kunstst., 56, p.114 (2003).

4. Machado, A. V. \& Van Duin, M. - Polymer, 46, p.6575 (2005). http:// dx.doi.org/10.1016/j.polymer.2005.05.011

5. Coran, A. Y. - "Thermoplastic Elastomeric Rubber - Plastic Blends", in: Handbook of Elastomers, cap 10, A. K. Bhowmick; H. L. Stephens (ed.), Marcel Dekker Inc. New York (2001).

6. Jacobi, M. M.; Schneider, L. K.; Freitas, L. L. \& Schuster, R. - Kautsch. Gummi Kunstst., 59, p.49 (2006).

7. Nicolini, A. ; Rocha, T. L, A. C. \& Jacobi, M. A. M. - J. Elast. Plast., 41, p.433 (2009). http://dx.doi.org/10.1177/0095244309100531

8. Naskar, K. - Rubber Chem. Technol., 80, p.504 (2007). http://dx.doi. org/10.5254/1.3548176

9. Martin, G.; Barres, C.; Sonntag, P. Garois, N. \& Cassagnau, P. - Eur. Polym. J., 45, p.3257 (2009). http://dx.doi.org/10.1016/j. eurpolymj.2009.07.012

10. Wanga, Z.; Chenga, X. \& Zhao, J. - Mater. Chem. Phys., 126, p.272 (2011).

11. Brostow, W.; Datashvili, T. \& Hackenberg, K. P. - Polym. Compos., 31, p.1678 (2010). http://dx.doi.org/10.1002/pc.20958

12. Ferro, S. - Plast. Mod., (343) (2003).

13. Trifkovic, M.; Sheikhzadeh, M.; Choo, K. \& Rohani, S. - J. Appl. Polym. Sci., 118, p.764 (2010).

14. Coran, A. Y. \& Patel, R. - Rubber Chem. Technol., 53, p.781 (1980).

15. Kresge, E. N. - Rubber Chem. Technol., 64, p.469 (1991). http://dx.doi. org/10.5254/1.3538564

16. Coran, A. Y.; Patel, R. \& Williams-Headd, D. - Rubber Chem. Technol., 58, p.1014 (1985). http://dx.doi.org/10.5254/1.3536097

17. Mehrabzadeh, M. \& Delfan, N. - J. Appl. Polym. Sci., 77, p.2057 (2000). http://dx.doi.org/10.1002/1097-4628(20000829)77:9\%3C2057::AIDAPP23\%3E3.0.CO;2-8

18. Kumar, C. R.; Sobha, V.; Nair, K.; George, E.; Oommen, E. \& Thomas, S. - Polym. Eng. Sci., 43, p 1555. (2003). http://dx.doi.org/10.1002/ pen. 10131

19. Chowdhury, R.; Banerji, M. S. \& Shivakumar, K. - J. Appl. Polym. Sci., 100, p.1008 (2006). http://dx.doi.org/10.1002/app.22876

20. Chowdhury, R.; Banerji, M. S. \& Shivakumar, K. - J. Appl. Polym. Sci., 104, p.372 (2007). http://dx.doi.org/10.1002/app.24858

21. Gomes, A. C. O.; Soares, B. G.; Oliveira, M. G. \& Paranhos, C. M. Polímeros, 19, p.196 (2009).

22. l'Abee, R. M. A.; Van Duin, M.; Spoelstra, A. B. \& Goossens, J. G. P. - Soft Matter., 6, p.1758 (2010).

23. Babu, R. R.; Singha, N. K. \& Naskar, K. - J. Polym. Res., 17, p.657 (2010). http://dx.doi.org/10.1007/s10965-009-9354-z

24. Babu, R. R.; Singha, N. K. \& Naskar, K. - J. Appl. Polym. Sci., 117, p.1578 (2010).

25. Grima, M. M. A.; Talma, A. G.; Datta, R. N. \& Noordermeer, J. W. M. - Rubber Chem. Technol., 79, p.694 (2006). http://dx.doi. org/10.5254/1.3547961
26. Orza, R. A.; Magusin, P. C. M. M.; Litvinov, V. M.; Van Duin, M. \& Michels, M. A. J. - Macromolecules., 42, p.8914 (2009). http://dx.doi. org/10.1021/ma9016482

27. Naskar, K. \& Noordermeer, J. W. M. - Rubber Chem. Technol., 77, p.955 (2004). http://dx.doi.org/10.5254/1.3547862

28. Fagundes, E. C. M. \& Jacobi, M. A. M. - "Influência do Sistema de Reticulação sobre as Propriedades de TPVs a base de PA-6/NBR", in: Anais do $10^{\circ}$ Congresso Brasileiro de Polímeros, p 89, Foz do Iguaçu - PR, outubro (2009).

29. Fagundes, E. C. M.; Jacobi, M. A. M. - "High-Performance TPVs based on PA6/NBR", in: International Rubber Conference - IRC, Nuremberg, Alemanha (2009).

30. American Society for Testing and Materials - ASTM. - "ASTM D412: Standard Test Methods for Vulcanized Rubber and Thermoplastic Elastomers-Tension".

31. American Society for Testing and Materials - ASTM. - "ASTM D395: Standard Test Methods for Rubber Property-Compression Set".

32. American Society for Testing and Materials - ASTM. - "ASTM D471: Standard Test Method for Rubber Property-Effect of Liquids".

33. Saleesung, T.; Saeoui, P. \& Sirisinha, C. - Polym. Test., 29, p.977 (2010). http://dx.doi.org/10.1016/j.polymertesting.2010.08.008

34. Soni, R. K.; Singh, H.; Dutt, K. \& Arora, P. - J. Polym. Res., 17, p.411 (2010). http://dx.doi.org/10.1007/s10965-009-9327-2

35. Thitithammawong, A.; Nakason, C.; Sahakaro, K. \& Noordermeer, J. W. M. - J. Appl. Polym. Sci., 111, p.819 (2009).

36. Babu, R. R.; Singha, N. K. \& Naskar, K. - J. Polym. Res., 18, p.31(2011). http://dx.doi.org/10.1007/s10965-010-9388-2

37. Tang, X. G.; Yang, W.; Shan, G. F.; Xie, B. H.; Yang, M. B. \& Hou, M. - J. Polym. Sci. Part B: Polym. Phy., 47, p.912 (2009). http://dx.doi. org/10.1002/polb.21698

38. Chapman, A. V. \& Tinker, A. J. - Kautsc. Gummi Kunstst., 56, p.533 (2003).

39. Coran, A. Y. \& Patel, R. - Rubber Chem. Technol., 53, p.141 (1980). http://dx.doi.org/10.5254/1.3535023

40. Coran, A. Y. \& Patel, R. - "Thermoplastic Elastomers Based on Dynamically Vulcanized Elastomer-Thermoplastic Blends", in: Thermoplastic Elastomers: a comprehensive review, cap 7, N. R. Legge, G. Holden, H. E Schoreder (eds.), Hanser Publishers, Nova York (1987).

41. Radusch, H. J. - "Phase Morphology of Dynamically Vulcanized Thermoplastic Vulcanizates", in: Micro-and nanostrutured multiphase polymer blend systems: phase morphology and interfaces, cap.9, C. Harrats, S. Thomas, G. Groeninckx (eds.), CRC Press Taylor\&Francis, Boca Raton (2006).

42. Koning, C.; Van duin, M.; Pagnoulle, C. \& Jerome, R. - Prog. Polym. Sci., 23, p.707 (1998). http://dx.doi.org/10.1016/S00796700(97)00054-3

43. Antunes C. F.; Machadoa, A. V. \& Van Duin, M. Morphology development and phase inversion during dynamic vulcanisation of EPDM/ PP blends. Eur Polym J, 47, p.1447 (2011). http://dx.doi. org/10.1016/j.eurpolymj.2011.04.005

44. Babu, R. R.; Singha, N. K. \& Naskar, K. - Polym. Bull., 66, p.95 (2011). http://dx.doi.org/10.1007/s00289-010-0328-4

45. Chauhan, K. D.; Soni, R. K. \& Singh, H. - Int. J. Plast. Technol., 14, p.117 (2010). http://dx.doi.org/10.1007/s12588-010-0016-0

Enviado: $21 / 03 / 11$

Reenviado: $30 / 06 / 11$ Aceito: 07/08/11 